\title{
Haematological Hoax, Hokum or Hobson's Choice? Bechamp Versus the Rest of the World!
}

\author{
Seun Ayoade* \\ BSc (Hons) Physiology, University of Ibadan, Nigeria
}

Received: 閏July 05, 2018; Published: 笽July 12, 2018

*Corresponding author: Seun Ayoade, BSc (Hons) Physiology, University of Ibadan,P.O. Box 22325, OyoState, Nigeria

\section{Introduction}

Virtually everybody on this planet has had the unfortunate experience of bleeding at least once. Whether mistakenly cutting oneself while shaving, or being punched in the face, or nicking a finger while using a blade or knife- we have bled. Blood flowed from the injured body part for a while and then stopped. Why? If a bucket or bottle of water is punctured it leaks till it becomes empty. Not so with the human body. Blood clots. How does this happen? The germ theory (which denies the existence of the microzymas) and the terrain theory/germ terrain duality theory (which do not) have radically varying views about how this phenomenon occurs.Around the world today, in almost all medical schools the germ theory version of blood clotting is taught. There is a different version-one that was discovered and elucidated by Professor Antoine Pierre Bechamp over a century ago.You have probably never heard this version of blood clotting. It literally is Bechamp against the rest of the world!

\section{Conventional Theory of Blood Clotting [According to Germ Theory][1]}

a. Blood clotting occurs through the chemical process of coagulation or haemostasis

b. Blood [i.e. roughly in its entirety] clots.

c. When a small blood vessel is transacted or damaged the injury initiates a series of events that leads to the formation of a clot (haemostasis). This seals off the damaged region and prevents further blood loss. The initial event is constriction of the vessel and formation of a temporary haemostatic plug of platelets that is triggered when platelets bind to collagen. This is followed by the conversion of the plug to the definitive clot. The in vivo action of the clotting mechanism is balanced by limiting reactions that normally prevent clots from developing in uninjured vessels and maintain the blood in a fluid state. It is worth emphasizing that a balance between many complex, interrelated systems must be maintained to prevent haemorrhage while preventing intravascular coagulation. The factors involved include the endothelium of the blood vessels and the collagen underlying it, vascular tone, the platelets, the clotting and fibrinolytic systems, and the flow characteristics of blood within the blood vessels.

d. The constriction of an injured arteriole or small artery may be so marked that its lumen is obliterated. The vasoconstriction is due to serotonin and other vasoconstrictors liberated from the platelets that adhere to the walls of the damaged vessels. It is claimed that for a time after being divided transversely, arteries as large as the radial artery constrict and may stop bleeding. However, this is no excuse for delay in ligating the damaged vessel. Furthermore, arterial walls cut longitudinally or irregularly do not constrict in such a way that the lumen of the artery is occluded, and bleeding continues.

e. The loose aggregation of platelets in the temporary plug is bound together and converted into the definitive clot by fibrin. The clotting mechanism responsible for the formation of fibrin involves a cascade of reactions in which inactive enzymes are activated, and the activated enzymes in turn activate other inactive enzymes. The complexity of the system has in the past been compounded by variations in nomenclature, but acceptance of a numbering system for most of the various clotting factors has simplified the situation.

f. The fundamental reaction in the clotting of blood is the conversion of the soluble plasma protein fibrinogen to insoluble fibrin. The process involves the release of two pairs of polypeptides from each fibrinogen molecule. The remaining portion, fibrin monomer, then polymerizes with other monomer molecules to form fibrin. The fibrin is initially a lose mesh of interlacing strands. It is converted by the formation of covalent cross linkages to a dense, tight aggregate. This latter reaction is catalysed by activated factor XIII and requires $\mathrm{Ca} 2+$.

g. The conversion of fibrinogen to fibrin is catalysed by thrombin. Thrombin is a serine protease that is formed from its circulating precursor, prothrombin by the activation of activated factor $\mathrm{X}$. factor $\mathrm{X}$ can be activated by reactions in either of two systems, an intrinsic and an extrinsic system. 
h. The initial reaction in the intrinsic system is conversion of inactive factor XII to active factor XII [XIIa]. This activation, which is catalysed by high molecular weight kininogen and kallikrein can be brought about invitro by exposing the blood to electronegatively charged wettable surfaces such as glass and collagen fibres. Activation in vivo occurs when blood is exposed to the collagen fibres underlying the endothelium in the blood vessels. Active factor XII then activates active factor XI, and active factor XI activates active factor IX. Activated factor IX forms a complex with active factor VIII, which is activated when it is separated from von Willebrand factor. The complex of IXa and VIIIa activate factor X. phospholipids from aggregated platelets (PL) and $\mathrm{Ca} 2+$ are necessary for full activation of factor $\mathrm{X}$. the extrinsic system is triggered by the release of tissue thromboplastin, a protein-phospholipid mixture that activates factor VII. The tissue thromboplastin and factor VII activate factors IX and X. in the presence of PL, Ca2+ and factor $\mathrm{V}$, activated factor $\mathrm{X}$ catalyses the conversion of prothrombin to thrombin.

\section{Professor Be champ's Version of Blood Clotting[2]}

a. Blood clots through the chemical process of fermentation.

b. The entire blood does not really coagulate. What we have is an ILLUSION of blood coagulating. What actually happens is that the blood ferments due to the action of microzymas.

c. The blood really contains three kinds of anatomical elements: the red globules, the white globules and the microzymian molecular granulations. The three sorts of anatomical elements are living, in so much as they are organized and contain microzymas which I have proved to be living by their function as ferments and by their capacity to become vibrionens by individual evolution, which was a novelty for physiologists and even for chemists.

d. But it is also necessary to understand in another way than has heretofore been done the meaning of what is called the coagulation of the blood. In truth, THE BLOOD DOES NOT COAGULATE. The experiment proves it; it is the substance of the atmosphere of the third anatomical element of the flowing issue which, in undergoing the allotropic change of coagulation, gives to the aggregate of the phenomenon the appearance of a total coagulation; but, as we have seen, it is only an illusion. The supposed spontaneous coagulation of the blood is at bottom, only the end of the first phase of the spontaneous alteration of the flowing tissue, as the cadaveric rigidity, marks the first phase of the spontaneous alteration of the muscular tissue.

e. What is called the phenomenon of the spontaneous coagulation of the blood is not at all a coagulation of the blood itself, but that of a portion of its third anatomical element. It will then clearly appear, that that which is improperly called a coagulation is only the first phase of a much more complete alteration of the blood, involving the destruction of its blood globules and other changes, even of that of its red colouring matter; and further that this spontaneous alteration of the blood is but a special case of a very general phenomenon, that of the spontaneous alterability of all animal matter, solid or humoral, abstracted from an animal, whether living or dead; an alterability, physiologically spontaneous, necessary, drawing with it the destruction even of the cellular anatomical elements themselves, as the consequence of phenomena of FERMENTATIONS OF A SPECIAL KIND, whereof the MICROZYMAS of these matters are the PRINCIPAL AGENTS.

f. There is to be found, says M. Frey, in human blood besides globules, agglomerations of small pale granulations 0.001 millimetre to 0.002 millimetre in diameter (Schultz); adding that these granulations, which had been noticed before, showed themselves sometimes with active movements of protoplasm, sometimes with a molecular movement (Brownian movement).

g. Further these molecular granulations have been observed in other humours and animal tissues and many opinions had been expressed as to their role, but it was not known what that role was, nor whether they were organized.

h. A microzyma, (whose diameter at most is 0.0005 millimetre) being so very small becomes visible when the albuminoid matter of the enveloping atmosphere, outside of the vessel, begins to undergo the allotropic modifications which cause it to acquire the properties which it possesses in the fibrin. Transparent crystalline is anatomically constituted by two layers of crystalline tubes. Further it contains, like other anatomical elements, a crowd of microzymas. All this the microscope is incapable of showing directly because all the parts have in the entire organ the same refractive index. But so soon as, by grinding, this organization is destroyed, the conditions of the existence of the anatomical elements of the organ being altered, microzymas and crystalline tubes become visible.

i. The microzyma, enveloped in the atmosphere of a special albuminoid substance, insoluble in the sanguineous medium I have named "Haematic Microzymian Molecular Granulation".

j. And now, when one considers that the weight of these microzymian molecular granulations, deduction made of the molecular granulatons which are furnished by the same volume of defribrinated blood, represents very nearly the weight of the fibrin obtained from the same volume of blood by whipping, it becomes evident that ordinary fibrin is nothing else than the microzymian granulations, heaped up and soldered together, whose albuminoid atmosphere has undergone, outside the vessel, an allotropic modification, by virtue of which, from having been directly soluble in very dilute hydrochloric acid, it has become soluble in it, only as a function of time and of 
temperature. The foregoing destroys the hypothesis of the plasma and verify, while completing, the conceptions of Hewson, of Milne-Edwards, of J.B Dumas, regarding the existence of the fibrin in the condition of fine granulations in the blood.

k. The coagulation being complete, by degrees the clot divides itself spontaneously into two parts. That which, in the clot, encloses the globules; that is to say the network of fibrin formed by the soldering of the microzymian molecular granulations, contracts then more and more, up to a certain limit, preserving the shape of the vessel in which the clot is moulded, and while the retraction takes place a part of the intercellular liquor is expelled, constituting what is called the serum, in which the retracted tissue is now immersed.

l. And the first serum thus expelled is transparent and lemon coloured, but by degrees the oxygen which the intercellular liquor holds dissolved, as well as that which the globules contain, is consumed; then is manifested the phenomenon observed and explained by J.B. Dumas in globules deprived of oxygen; they change, and their changed colouring matter is diffused in the circumambient serum which becomes more and more of a deep red colour. This is what the plasmatic hypothesis cannot explain, if one regards the plasma as a liquid in which all the components are in perfect solution. m. It is time to erase from the language of science the words 'plasma', 'plasmin', 'fibrinogen' and of 'fibrinoplastic' with which it has been encumbered. There must also be erased for the explanation of the phenomenon the pretended influence of the globules, of calcareous or other salts, catalytic actions of contact etc.-not to speak of various occult influences. An exact knowledge of the anatomy of the blood and of the conditions of existence of the anatomical elements will suffice.

n. The physiological theory of the spontaneous coagulation of the blood, founded on the existence of a third anatomical element, the microzymian molecular granulations of the flowing tissue, sufficiently explains the facts.

\section{Conclusion}

The bottom line is that what we are being taught about blood clotting is not the truth, the whole truth and nothing but the truth. Or vice versa. One of these theories is a partial or complete hoax.

\section{References}

1. WF Ganong (1998) Review of Medical Physiology Lange Medical Books, (18 ${ }^{\text {th }}$ Edn.); pp. 505-506.

2. A Bechamp (1991) Blood and Its Third Anatomical Element Kessinger Legacy Reprints pp. 27-156.

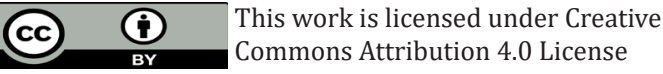

To Submit Your Article Click Here: Submit Article

DOI: $10.32474 /$ PRJFGS.2018.01.000120 